\title{
(In)escapable Affect? Exploring Factors Influencing Privacy-Related Behavioral Intentions
}

\author{
Agnieszka Kitkowska \\ Karlstad University \\ agnieszka.kitkowska@kau.se
}

\author{
Erik Wästlund \\ Karlstad University \\ erik.wastlund@kau.se
}

\author{
Leonardo A. Martucci \\ Karlstad University \\ leonardo.martucci@kau.se
}

\begin{abstract}
The study was run to investigate exploratory capabilities offactors such as individual characteristics, privacy concerns and information disclosure in the context of privacy behaviors. The research examined whether affective states arising from immediate emotions alter such capabilities. The results of an online study with 474 international participants demonstrate that immediate emotions might influence information sharing. The effect of privacy concerns, personality and information disclosure on the willingness to share is stronger when participants are in a neutral affective state. However, when the positive or negative feelings take over, the influence of these factors on willingness to share decreases. In this article, we postulate the necessity to include immediate emotions into research on privacy-related decision-making and discuss the applicability of our results in the context of privacy UIs.
\end{abstract}

\section{Introduction}

Increasing dependence on Internet-connected devices results in an extensive data collection. Online companies possess large amounts of data about individuals, ranging from behavioral, economical to very private health information. Such data is collected by, shared with or sold to unknown third parties or collaborating organizations. As a result, individuals are at increased risk to severe privacy violations (e.g., economic loss, reduced autonomy or psychological damages).

The emerging privacy concerns lead policymakers to apply measures diminishing the risks of over-disclosures. For instance, in Europe, the General Data Protection Regulation (GDPR) requires online service providers to ensure that users are aware of data provision practices. Hence, researchers have been developing and testing different visual designs to enhance privacy awareness $[1,2,3]$. The design of such privacy user interfaces (UI) require an advanced knowledge of how people decide on their privacy.

This research aims to gain such insights by investigation of the role of individual characteristics and affective states in privacy-related decision-making. Specifically, the goal of this work is to examine how affective states, together with other factors, influence the willingness to share information.

The motivation for our research design originates mostly in behavioral sciences and psychology. Past work shows that visual stimulus, thorough elicitation of emotional responses, impacts people's attitudes and behaviors [3]. Hence, such affective incentives are often applied in digital designs. For instance, aesthetically pleasing web applications, which target people's emotions, aim to enhance engagement and product experience [4]. Feelings resulting from interactions are crucial for the design, and past research showed that they are inevitable for cognitive processing, construction of preferences or motivations $[5,6,7,8]$.

This paper is structured as follow. Section 2 presents an overview of related work and introduces research questions. Section 3 details methods used to build the experiment. Section 4 presents the results of statistical analysis. Section 5 discusses findings, their implications and study's limitations. Concluding remarks are presented in Section 6.

\section{Related Work}

Decision-making might be described as making a choice, or choices from several options [9]. However, this definition simplifies the issue. In reality, decisions vary in complexity. The most common theories adapted to investigate the decision-making process come from economics. Conservative economic approach assumes that choice is based on rational calculations aiming to, e.g., maximize benefits. Nevertheless, past research showed that the economic approach might be insufficient due to various intrinsic and extrinsic factors influencing cognitive processing [10].

People's decisions are affected by psychological 
biases, e.g., optimism bias (people make a decision, they are confident that certain risks will not happen to them), hyperbolic discounting (tendency to value present, smaller rewards more than future, larger rewards), bounded rationality (cognitive limitations) [11]. Further, people use mental shortcuts, i.e., simple heuristics $[10,11]$. These enable quick and automatic choices, as opposed to effortful evaluations based on slower and analytic calculations [10].

Another aspect increasing the complexity of decision-making processes is a matter of subjective evaluations. For instance, decisions of others might seem illogical or irrational; yet, they are justified by the decision-maker. This behavior might be explained with individual characteristics, such as personality differences. In psychology, such features are found to remain relatively stable over time and, depending on situational factors, to shape behavior [12].

\subsection{Decision Making and Affect}

To discuss the role of affect we must first define related terminology. Despite a considerable interest received by social sciences, there is not much consensus regarding the definition and differentiation between affect and emotion. In this work, we follow description presented in $[13,14]$ that define the affect as general feelings; as "the superordinate umbrella of constructs that involves emotion, mood, and emotion-related traits" [14].

Past findings show that subjective evaluations frequently depend on the affect heuristic, emotional state consciously or unconsciously impacting behavior $[15,16]$. In recent research, affect was recognized as an essential factor in guiding people's decisions. For instance, the risk as feelings hypothesis proposes that behavior is influenced by cognitive evaluations, as well as feelings [17, 18]. Similarly, the affect-as-information theory suggests that affect is present during the assessment of an object's value [19].

In this work, we adapt the concept proposed by Loewenstein and Lerner, presenting two ways in which affect may impact behavior [13]. The first is through expected emotions, emotionally charged predictions of outcomes. The second is immediate emotions, which are the subject of this study. Immediate emotions can influence, both directly or indirectly, the decision. Their impact on choice depends on emotions' intensity. In the direct relationship, emotions do not affect all decisions, but only the ones relevant to the emotional state. They play an advisory role in the decision-making process. In our study, we focus on the indirect (incidental) effects of immediate emotions, i.e., emotions that are caused by accidental influences unrelated to the decision.

2.1.1. Affect in Privacy-Related Research. One of the more influential frameworks presenting privacy-related decision-making is the APCO model (Antecedents $\rightarrow$ Privacy Concerns $\rightarrow$ Outcomes) [20]. The model postulates that privacy behavior is preceded by privacy concerns, their antecedents (e.g., personality, previous experience), trust and risk-benefit calculation. The revised version of the APCO includes two systems responsible for cognitive processing: effortful and effortless [21]. The inclusion of two systems of reasoning implies that psychological biases, as well as affect heuristic moderate privacy attitude and behavior.

Past work showed that affect subconsciously shapes privacy risk perceptions [22]. The relationship between decision-making and feelings was frequently framed negatively, implying that affect builds a premise for irrationality. For instance, the imbalanced emotional state may lead to an increased level of information disclosure [22]. On the other hand, researchers demonstrate that initial emotions have a lasting impact on privacy beliefs, for example, in an e-commerce environment [23]. Others show how affective states can reveal privacy attitudes. Coopamootoo et al. examine the role of fear and happiness concerning privacy or sharing attitude [24]. According to their findings, negative valence increases privacy attitude and decreases sharing, while positive valence increases sharing attitude and decreases privacy attitude.

Following concepts of Loewenstein and Lerner [13], and the APCO model [21] this work aims to advance research on the role of affect in privacy decisions. Hence, our first research question is:

RQ1: Do affective state influences the willingness to share information?

2.1.2. Privacy Attitudes and Behaviors. According to the APCO framework, behavioral reactions, such as information disclosure, are outcomes of the relationship between privacy concerns and their antecedents [20]. Behavioral outcomes differ due to many factors, e.g., risk perceptions, psychological states, trust or individual characteristics [25, 26, 27, 28, 29].

In this study, to improve understanding of privacy attitude-behavior relationship, we investigate how privacy concerns and the amount of disclosed information influence the willingness to share. We interpret sharing as a behavioral outcome, e.g., 


\begin{tabular}{|c|c|c|c|c|c|}
\hline $\begin{array}{l}\text { Demographic } \\
\text { characteristics }\end{array}$ & $\begin{array}{l}\text { Personality } \\
\text { Traits }\end{array}$ & $\begin{array}{c}\text { Affect eliciting } \\
\text { video }\end{array}$ & $\begin{array}{c}\text { Information } \\
\text { disclosure }\end{array}$ & $\begin{array}{c}\text { Information } \\
\text { sharing }\end{array}$ & $\begin{array}{c}\text { Emotion } \\
\text { measurement }\end{array}$ \\
\hline
\end{tabular}

Figure 1: Order of the experiment's main sections/questionnaires.

when people give their consent to future information processing [30]. Past work concentrates on sharing of different types of personal information (e.g., sensitive or non-sensitive) with various recipients (e.g., third parties, friends) in many contexts (e.g., social media, location) [31, 32, 33, 32, 34]. To advance past findings, we ask the following research question:

RQ2: Do the influence of information disclosure and privacy concerns on the willingness to share differ among participants exposed to different affective stimulus?

\subsection{Decision-Making and Personality}

Loewenstein and Lerner proposed that individual characteristics, such as personality, precede decisions [13]. In the field of privacy, personality differences have been recognized as factors impacting attitudes [20]. Some of the past studies acknowledge personality traits as antecedents of privacy concerns and indirect influencers on behavior [35]. For instance, Junglas and Spitzmüller found that agreeableness, conscientiousness, and imagination contribute to the formulation of "Concerns for Privacy" (CFP) [36]. Further, past research shows a potential influence of personality traits on information disclosure; e.g., an openness impacting social media disclosure [37]. On the other hand, studies indicate that the five-factor personality model is not the strongest predictor of privacy attitudes when compared to, e.g., risk-taking [38].

To confirm previous findings, we investigate the role of The Big Five personality traits (extraversion, agreeableness, conscientiousness, neuroticism, openness to experience (imagination) ) [39]. Hence, we propose the following research questions:

RQ3: Is there a relationship between The Big Five personality traits, information disclosure and willingness to share?

RQ4: Do the relationship between The Big Five personality traits and willingness to share differ among participants exposed to a particular affective stimulus?

\section{Methods}

To address our research questions, we conducted an online experiment. We applied a between-subjects design (three groups). The independent variables in the study were privacy concerns, information disclosure, affective state elicited with videos (negative $\rightarrow$ neutral $\rightarrow$ positive) and five personality traits; the dependent variable was the willingness to share information. The overview of the study is presented in Figure 1.

The research received ethical approval from the Swedish Ethical Review Agency. When possible, we applied data anonymization processes to decrease risks to research participants. All respondents that completed the study received payment ranging from $\$ 1.50-\$ 2.50$, depending on their country of residence, to make it equivalent of local minimum salary. The average completion time was 8.5 min.

\subsection{Participants and Data Preparation}

We collected participants through crowd-sourcing platforms, MTurk and Clickworkers. Different geographic regions were selected to ensure variability of the sample and control for potential cultural dependencies.

Overall, we collected 543 responses. All incomplete responses were removed, and the time relative speed index method was used to clean the data [40]. Additionally, all cases where participants failed video checks (i.e., misidentified characters in a video, stated that they closed their eyes or turned away from the screen while watching a video) were discarded, reducing the sample size to 515 . Lastly, cases with equal levels of positive, neutral and negative, self-reported affective states (means +/ - half SD) and outliers were removed. The final number of participants equalled 474 .

\subsection{Pilot and Manipulation Checks}

We designed a pilot study to check the manipulation method and study design. After evaluating the pilot study, we applied appropriate changes and ran the final experiment.

After completion of the final experiment, One-Way ANOVA was used to check whether the videos elicited affective states: positive, neutral and negative. The Levene's test was significant $(p<.05)$, implying that the assumption of homogeneity of variances was violated. Hence, we used Welch ANOVA when 


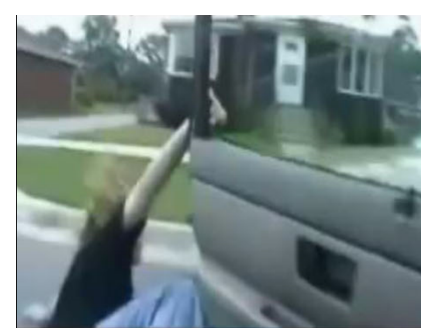

(a)

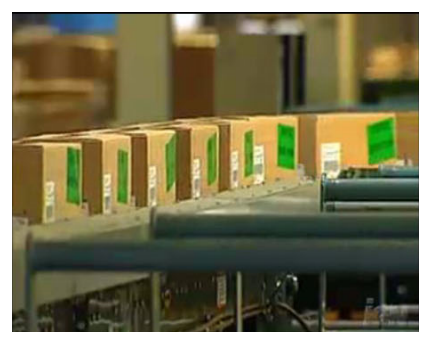

(b)

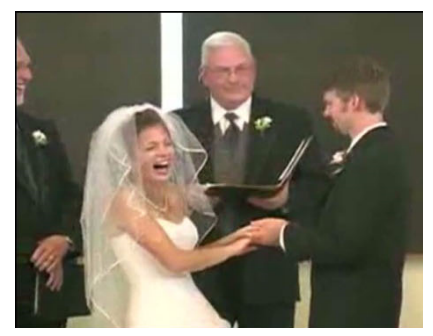

(c)

Figure 2: Thumbnails from the emotion eliciting videos: (a) Negative, (b) Neutral, (c) Positive.

appropriate. The results were significant, indicating difference between the experimental groups in their affective states: positive $F(2,282.8)=362.9, p<$ .001 , neutral $F(2,289.8)=168.2, p<.001$, and negative $F(2,289.8)=176.8, p<.001$. The Tukey and Games-Howell (for non-equal variances) post-hoc tests clarified the differences. Respondents who watched the positive video reported significantly higher positive than a negative or neutral affective state, $p<.001$. Participants who watched the neutral video reported significantly higher neutral than the positive or negative state, $p<.001$. Lastly, people who watched the negative video reported significantly higher negative than positive or neutral affect, $p<.05$.

\subsection{Demographics}

We collected basic demographic characteristics, such as age, gender, education and nationality. The study respondents were from the USA (41\%), UK (23\%), Scandinavia (17\%) and Germany (19\%). All respondents were over 18 years old, with 195 females and 279 males. The participants' educational level varied from basic schooling (high school) to higher education (Ph.D. or higher). Table 1 presents detailed demographics.

\subsection{Instruments Applied in the Study}

Our study contained instruments measuring The Big Five personality traits, affect-eliciting videos (approx. $60 \mathrm{sec}$ ), information disclosure, willingness to share and privacy concerns. Figure 1 presents the order the study was performed in.

3.4.1. The Big Five Personality Traits. To assess The Big Five personality traits, we applied the Mini IPIP scale acquired from Donnellan et al. [41]. It is a short instrument validated in previous research, preventing participants' cognitive over-workload [42].
Table 1: Demographics.

\begin{tabular}{llll}
\hline \multirow{2}{*}{ Gender } & & Frequency & Percent \\
\hline \multirow{2}{*}{ Countries } & Memale & 195 & 41 \\
& USA & 279 & 59 \\
& UK & 198 & 41 \\
& Scandinavia & 108 & 23 \\
Age & Germany & 87 & 17 \\
& $18-24$ & 77 & 19 \\
& $25-34$ & 174 & 16 \\
& $35-44$ & 122 & 26 \\
& $45-54$ & 69 & 14 \\
& over 55 & 32 & 7 \\
\hline \multirow{6}{*}{ Education } & Basic & 116 & 25 \\
& Some college & 71 & 15 \\
& Professional & 72 & 15 \\
& Bachelor & 152 & 32 \\
& Master \& higher & 63 & 13 \\
\hline
\end{tabular}

The scale consists of 20 statements, and each personality trait is assessed with four items (scored on the scale ranging from 1- "Very inaccurate" to 5 - "Very accurate"). To compute the results, we followed a recommendation from the International Personality Item Pool website and summed the scores (https://ipip.ori.org/). As a result, the scores for each trait ranged from 0 to 20 . We ran the Principal Component Analysis (PCA) and checked whether all items load correctly. One of the characteristics did not load appropriately (imagination); hence, we removed it from further analysis.

When checking psychometric scales, two elements should be considered: reliability and validity. Validity concerns the relationship between concept and criterion. We checked the concurrent validity and assessed the instrument's reliability with Cronbach's $\alpha$ (Table 2). 
Table 2: Cronbach's $\alpha$ for personality traits; $\mathrm{N}=474$.

\begin{tabular}{llll}
\hline Personality trait & $\alpha$ & $M$ & $S D$ \\
\hline Extraversion & .85 & 11.71 & 1.73 \\
Agreeableness & .81 & 12.49 & 1.76 \\
Conscientiousness & .72 & 11.62 & 1.85 \\
Neuroticism & .80 & 11.90 & 1.90 \\
\hline
\end{tabular}

The recommended Cronbach's alpha ranges from .7 to .9 , hence we accepted personality scores [43].

3.4.2. Emotion Elicitation. Past research identified various methods eliciting affective states, for instance, text descriptions, images, videos, self-reflection [44]. In this study, we used videos, since this form of elicitation has been proven effective [45, 46].

Our study contained short videos, which aimed to elicit positive, neutral (control) and negative affective states (Figure 2). All videos were acquired from past research performed at Stanford University [47]. From over 200 amateur short video clips, the videos with the highest scores in emotion elicitation were selected. The chosen clips were combined in approximately 60 second long videos. The participants were randomly assigned to video groups. The study included checks to assess whether the respondents had watched the videos.

We assessed emotional valence by asking participants to what extent they felt negative, neutral or positive while watching the videos. Assessment scores were collected on a visual analogue scale ranging $0-100$ ("I have not felt it at all" - "I have strongly felt it" respectively).

3.4.3. Information Disclosure Scale. Half of the items used to build a scale measuring information disclosure were acquired from an instrument validated in past research [48]. The other half was created for this study, ensuring the same format. Overall, the scale consisted of eight items measuring sensitive information disclosure, including questions of personal nature, such as the number of sexual partners, name, salary, and more. Each question contained the answer "I prefer not to say". The factual answers were not collected to maintain the respondent's privacy. Instead, the dichotomous data were gathered: 1 if the participant disclosed information, 2 if a participant selected "I prefer not to say". The scores were reversed and summed to compute the disclosure score.

The KR20 (Kuder and Richardson) estimate of reliability is recommended for dichotomous scales [49].
It is a particular case of Cronbach's $\alpha$, and has the same interpretation; hence, the Cronbach's $\alpha$ was used to check reliability. The Cronbach's $\alpha$ for information disclosure scale was good, equaling .77.

3.4.4. Willingness to Share. To measure the willingness to share, we used a scale consisting of four items. The respondents were asked whether they would allow us to share all, some or none of the information disclosed in the survey with third parties or on social media. Additionally, the participants were asked whether they would provide an email address so we could share it. The responses were summed, resulting in the lowest scores (min 4) indicating a low level of sharing and the highest ratings (max 11) indicating high sharing. The Cronbach's $\alpha$ was .85, confirming that the scale's reliability was good.

3.4.5. Privacy Concerns. We measured privacy harms concerns with a scale developed in previous research [50]. We modified some of the scale's items, hence, to ensure reliability and validity, we ran Exploratory Factor Analysis. The KMO was good, .904 , and Bartlett test of sphericity was significant, $p<$ .001. The results of the analysis did not match the original seven dimensions of privacy harms concerns. We re-run PCA. All factors had communalities $>.5$, and accounted for $62 \%$ cumulative variance explained. We applied Varimax rotation and extracted four factors: security concerns, unauthorised access, interrogation and distortion. All items, their loadings and Cronbach's $\alpha$ for each factor are presented in the Table 3 ).

\section{Results}

After checking the validity and reliability of instruments used to measure all latent concepts, we proceeded with further data analysis. To answer the research questions, we applied statistical tests.

\subsection{ANOVA}

To answer $R Q 1$ we ran One-Way ANOVA with video groups (negative, neutral, positive) as the independent variable and willingness to share information as the dependent variable. We checked the test's assumptions. Both the Levene's test for homogeneity of variance and equality of means was good. However, the resulting model was insignificant, $F(474,2)=1.102, p>.05$ indicating that the willingness to share does not differ between groups. 
Table 3: Privacy concerns scale. Results of Exploratory Factor Analysis.

\begin{tabular}{|c|c|c|c|c|}
\hline Item & 1 & 2 & 3 & 4 \\
\hline \multicolumn{5}{|l|}{ Security Concerns, $\alpha=.868$} \\
\hline $\begin{array}{l}\text { It concerns me when companies do not immediately inform me if my online account } \\
\text { was compromised }\end{array}$ & .822 & & & \\
\hline It bothers me if my true contact details are available to the public & .738 & & & \\
\hline $\begin{array}{l}\text { It concerns me that my online accounts could be compromised and my information } \\
\text { could be used against me }\end{array}$ & .724 & & & \\
\hline It concerns me when online companies systems are insecure & .718 & & & \\
\hline $\begin{array}{l}\text { It would bother me if somebody shared information about my grief on their social } \\
\text { network }\end{array}$ & 662 & & & \\
\hline $\begin{array}{l}\text { It concerns me that others may use information disclosed on my online profiles to } \\
\text { reach their own goals }\end{array}$ & .607 & & & \\
\hline $\begin{array}{l}\text { It bothers me if companies are allowed to collect information about my online } \\
\text { behavior, especially if it is linked to my online profiles }\end{array}$ & .594 & & & \\
\hline \multicolumn{5}{|l|}{ Unauthorized access, $\alpha=.828$} \\
\hline It bothers me that online medical service' employees can identify me & & .733 & & \\
\hline It bothers me that online companies' employees can see my name or address & & .647 & & \\
\hline $\begin{array}{l}\text { It bothers me that online services' employees may have access to my personal } \\
\text { information }\end{array}$ & & .646 & & \\
\hline $\begin{array}{l}\text { It concerns me that unauthorized person could access my online information and } \\
\text { threaten me }\end{array}$ & & .625 & & \\
\hline It worries me that the documents I store online could be used against me & & .606 & & \\
\hline \multicolumn{5}{|l|}{ Interrogation, $\alpha=.754$} \\
\hline It bothers me if online service providers contact me to collect my feedback & & & .781 & \\
\hline $\begin{array}{l}\text { It bothers me when I am receiving purchased products' review requests, so I rarely } \\
\text { respond to them }\end{array}$ & & & .753 & \\
\hline $\begin{array}{l}\text { It bothers me when online companies send online questionnaires or surveys, even if } \\
\text { they aim to improve services }\end{array}$ & & & .696 & \\
\hline \multicolumn{5}{|l|}{ Distortion, $\alpha=.678$} \\
\hline I am concerned what other people think of my online profiles & & & & .830 \\
\hline $\begin{array}{l}\text { I am concerned about my online reputation and to ensure my reputation I frequently } \\
\text { update my online profiles }\end{array}$ & & & & .772 \\
\hline It concerns me that others may misjudge me because of inaccurate online information & & & & .544 \\
\hline
\end{tabular}

\subsection{Correlations}

To examine the relationship between individual characteristics, willingness to share and information disclosure, we examined correlations (RQ3). After checking the test assumptions, Pearson's correlation was used to measure the relationship between variables. Table 4 presents correlations between variables. There are small, significant negative correlations between willingness to share and conscientiousness $(r=-.173$, $p<.001)$, and between willingess to share and neuroticism $(r=-.099, p<.05)$. There is also a significant negative correlation between information disclosure and conscientiousness $(r=-.112, p<.05)$, as well as positive correlation between disclosure and willingness to share $(r=.239, p<.001)$.

\subsection{Regression}

To answer the remaining research questions $(R Q 2$, $R Q 4)$ we ran regression analysis. We applied the split file procedure to compare three experimental groups, as per assignment to the video stimuli. First, we checked regression assumptions, such as linearity, multicollinearity, homoscedasticity. To check for linearity, we looked as scatterplots. To assess multicollinearity, we tested the tolerance values (all above .4) and VIF values (all between $>1$ and $<2.5$ ).

The applied split file procedure resulted in three regression models. The dependent variable in the 
Table 4: Pearson correlations. N=474; ** - significant at the level 0.001 (2-tailed); * - significant at the level 0.05 (2-tailed).

\begin{tabular}{lllllll}
\hline & EXTR & AGRE & CONS & NEUR & WILS & INFD \\
\hline Extraversion (EXTR) & 1 & & & & & \\
Agreeableness (AGRE) & $.131^{* *}$ & 1 & & & & \\
Conscientiousness (CONS) & -.005 & $.158^{* *}$ & 1 & & & \\
Neuroticism (NEUR) & .086 & .076 & $.136^{* *}$ & 1 & & \\
Willingness to share (WILS) & -.033 & -.056 & $-.173^{* *}$ & $-.099^{*}$ & 1 & \\
Information disclosure (INFD) & -.090 & -.084 & $-.112^{*}$ & .022 & $-.239^{* *}$ & 1 \\
\hline
\end{tabular}

Table 5: Results of regressions; ** - significant at the level 0.001 (2-tailed); * - significant at the level 0.05 (2-tailed).

\begin{tabular}{llllllllll}
\hline Variable & \multicolumn{4}{l}{ Negative $N=119$} & \multicolumn{3}{c}{ Positive $N=164$} & \multicolumn{3}{c}{ Neutral $N=191$} \\
\cline { 2 - 11 } & $\mathrm{B}$ & $\mathrm{SE} \mathrm{B}$ & $\beta$ & $\mathrm{B}$ & $\mathrm{SE} \mathrm{B}$ & $\beta$ & $\mathrm{B}$ & $\mathrm{SE}$ B & $\beta$ \\
\hline Security concern & -.037 & .019 & $-.231^{*}$ & -.044 & .017 & $-.263^{*}$ & -.048 & .016 & $-.286^{*}$ \\
Unauthorized access & .013 & .017 & .101 & .012 & .016 & .084 & .026 & .012 & $.204^{*}$ \\
Interrogation concern & -.022 & .012 & -.181 & -.008 & .010 & -.072 & -.022 & .008 & $-.201^{*}$ \\
Distortion concern & -.021 & .012 & -.175 & .006 & .011 & .051 & -.012 & .009 & -.100 \\
Information disclosure & -.474 & .158 & $-.264^{*}$ & -.197 & .129 & -.121 & -.438 & .119 & $-.237^{* *}$ \\
Conscientiousness & -.099 & .122 & -.070 & .217 & .118 & .146 & .316 & .090 & $.238^{*}$ \\
Extraversion & .139 & .134 & .095 & .004 & .118 & .002 & -.104 & .109 & -.063 \\
Agreeableness & -.090 & .124 & -.062 & .057 & .120 & .037 & .009 & .103 & .006 \\
Neuroticism & .290 & .122 & $.209^{*}$ & .011 & .113 & .007 & .079 & .095 & .054 \\
\hline
\end{tabular}

models was the willingness to share. The independent variables were privacy concerns (security, unauthorized access, interrogation, distortions), information disclosure and personality traits (conscientiousness, extraversion, agreeableness, neuroticism). All models resulted in a significant change in the $\mathrm{F}$ ratio $(p<.05)$.

In the negative group, concerns about security, information disclosure and neuroticism were found to statistically significantly affect willingness to share $(p<$ $.05)$. Overall model's predictive value was significant, $F(9,110)=3.879, p<.001, R^{2}=.241$. In the positive group security concerns were the only variable that was significant $(p<.05)$, with the overall model $F(9,156)=2.362, p<.05, R^{2}=.120$. Lastly, in the neutral group, there were five significant predictors of the willingness to share: concerns about security, unauthorized access, and interrogation, information disclosure and conscientiousness $(p<.05)$. The overall model value was $F(9,182)=7.698, p<.001, R^{2}=$ .276 . The detailed results of all three regressions are presented in Table 5.

\section{Discussion}

The main goal of this work was to examine the role of affect in privacy-related context. Specifically, we investigated whether there are differences in the willingness to share information among people influenced by immediate emotions. In this section, we discuss our findings and their potential implications.

Our results show that immediate emotions, causing affective state, on their own do not have a significant impact on the willingness to share. When considered as an independent variable, the effect of the negative, neutral and positive affective stimulus was not present. These findings conflict with the conceptual model proposed by Loewenstein and Lerner [14, 17]. Nonetheless, this result is not startling, considering that the affective states elicited in the study are not directly related to the decision at hand. Expectedly, the exploratory power of affect is weaker under such circumstances.

The results showed small but significant correlations confirming the relationship between some of the personality traits, information disclosure and willingness to share $(R Q 3)$. We found that conscientiousness and neuroticism have a negative relationship with the willingness to share. This result indicates a possible tendency that people who are more in control over their impulses, organized as well as nervous people, emotionally negative, seem to share less information. Additionally, as expected, people who disclose more information intend to share more information. Lastly, the correlation analysis 
demonstrated that participants with higher levels of conscientiousness appear to disclose less information.

Further, the regression analysis demonstrated that there are potential differences between participants assigned to different video groups when more "traditional" variables are set as independent factors (RQ2, RQ4). It seems that the most stable adjustor of behavioral intention is one of the privacy concerns, explicitly the concern about online security.

Among participants from the neutral group, the variables proposed in the APCO model, such as concerns, information disclosure and personality are the most effective predictors. For instance, concerns about security, unauthorized access, together with the information disclosure, conscientiousness and interrogation, significantly influence sharing. However, the impact of these variables differs among the participants assigned to negative and positive video groups.

It appears that conscientiousness does not significantly influence sharing when people are exposed to either positive or negative stimuli. Such finding corresponds to the revised APCO model, and some of the theories (e.g., affect-as-information) proposing that affect heuristics may take over rational calculations during the decision-making process. Let us consider conscientiousness. Per definition, it means control over impulses, facilitating a goal and task orientation [39], and could be interpreted as a trait supporting rational decision-making. Our results suggest that when a person is exposed to affective stimulus, the affective state may take over this trait, impacting behavioral outcome. It is possible, that positive or negative feelings trigger some of the psychological biases, decreasing risk perception and leading to greater information disclosure.

According to our results, the negative stimulus activated different personality trait: neuroticism. Such a conclusion is expected, as neuroticism is associated with nervousness, emotional negativity, relating to sadness, tension and anxiety. Our findings indicate that people with higher levels of neuroticism are under stronger influence of the negative stimulus and willing to share more information.

The most robust impact of the affect on willingness to share was found among the participants from the positive group. The regression analysis shows that in this group, only security concerns had a significant effect on the willingness to share. Both the conscientiousness and information disclosure had no significant impact on the outcome variable. Such finding indicates that, perhaps, regardless of the amount of disclosed information, positive feelings reduce careful information management and may lead to a higher willingness to share information. This result is in agreement with findings from [24].

\subsection{Additional Findings}

Beside answering research questions, this work confirms the effectiveness of affect elicitating method, such as the use of amateur videos. Our results demonstrate that manipulation worked at a highly significant level. In past work, privacy research about affect applied commercial videos, specially designed to influence viewers' emotional engagement. While such elicitation methods work, it might be difficult to convert them into visual representations of privacy issues, e.g., risks or harms. The amateur videos used in this study have a higher potential, as experts did not create them, and they represent real-life situations, which everyone can associate with.

Overall, our results show potential differences in information sharing among participants exposed to various affect-eliciting stimuli. We believe that our findings should be applied in future research examining models of privacy-related decisions, as they may help to improve models' predictable capabilities. Additionally, affect might be used in privacy UIs research, to investigate further whether the affect heuristics can indeed influence rational decision-making. For instance, knowledge about personality characteristics and privacy concerns could be applied to personalized privacy-enhancing technologies. Such PETs could include affective visual cues to test how the combined factors examined in our study influence privacy-related behaviors.

Additionally, our preliminary findings open a new way to advance research on the dark patterns. For example, future research could focus on privacy designs that elicit immediate emotions or on interfaces that include visual cues not related to privacy decisions. Future work could examine how affective UI elements unrelated to privacy influence user by unconscious elicitation of positive or negative emotional state.

\subsection{Limitations and Future Work}

Despite the experimental design applied in this study, our work is not free of limitations. Online research did not allow to collect observational data, such as reactions to visual stimuli. Additionally, emotional valence was measured in the form of self-reported assessment. Running the experiment in a lab could increase the reliability of the affective measurements. In the laboratory settings, researchers could record facial 
expressions, eye movements or other bodily responses. Further, affect was measured after the willingness to share, possibly causing ordering effect and influencing affective scores.

Our study did not contain privacy stimulus aiming to elicit affective response. Instead, we applied privacy-unrelated videos, which is the most substantial limitation of this study, as we could not conclude whether privacy stimulus would have the same effects. The study's ecological validity would increase if the experiment included visual representations of privacy issues (e.g., risks, harms, or display of privacy policies). All the above leave space for future research.

\section{Conclusion}

Online services require users to provide personal, often sensitive information. Every time people disclose such information, they make a privacy-related decision. Nevertheless, people are rarely aware of the potential consequences of disclosure. The ambiguity of privacy policies, consents and a lack of understanding of how the technology works, place people at risk. Although privacy concerned, people willingly or unwillingly over-disclose personal data. To improve the current landscape of personal information management and enhance privacy design, researchers and designers must gain an in-depth understanding of cognitive processes accompanying privacy decisions.

In this article, we advance knowledge about privacy-related decision-making. Specifically, we show that affective state resulting from immediate emotions may influence privacy-related behaviors. Our results demonstrate that stable factors, such as privacy concerns, personality traits and information disclosure impact willingness to share information. However, their role might be altered due to activation of affective states. Presented findings can be used as a theoretical foundation for further research.

\section{Acknowledgments}

This work has received funding from the European Unions Horizon 2020 research and innovation programme under the Marie Skłodowska-Curie grant agreement No 675730. For more information and access to the materials, please contact the authors.

\section{References}

[1] J. Angulo, S. Fischer-Hübner, T. Pulls, and E. Wästlund, "Usable Transparency with the Data Track - A tool for visualizing data disclosures," in Proceedings of the 33rd Annual ACM Conference on Human Factors in Computing Systems, pp. 1803-1808, 2015.
[2] B. P. Knijnenburg, "Privacy? I Can't Even! Making a Case for User-Tailored Privacy," IEEE Security and Privacy, vol. 15, no. 4, pp. 62-67, 2017.

[3] A. Acquisti, M. Sleeper, Y. Wang, S. Wilson, I. Adjerid, R. Balebako, L. Brandimarte, L. F. Cranor, S. Komanduri, P. G. Leon, N. Sadeh, and F. Schaub, "Nudges for Privacy and Security," ACM Computing Surveys, vol. 50, no. 3, pp. 1-41, 2017.

[4] L. Deng and M. S. Poole, "Affect in Web Interfaces: A Study of the Impacts of Web Page Visual Complexity and Order," MIS Quarterly, vol. 34, no. 4, pp. 711-730, 2010.

[5] D. A. Norman, Emotional Design. Why we love (or hate) everyday things. New York, USA: Basic Books, 2004.

[6] C. Wrigley, "Design dialogue: The visceral hedonic rhetoric framework," Design Issues, vol. 29, no. 2, pp. 82-95, 2013.

[7] G. L. Clore and A. Ortony, "Psychological construction in the OCC model of emotion.," Emotion review, vol. 5, no. 4, pp. 335-343, 2013.

[8] N. Schwarz, Feelings as information: Informational and motivational functions of affective states, pp. 527-561. New York, USA: The Guilford Press, 1990.

[9] G. Klein, "A naturalistic decision making perspective on studying intuitive decision making," Journal of Applied Research in Memory and Cognition, vol. 4, no. 3, pp. 164-168, 2015.

[10] D. Kahneman, "A Perspective on Judgement and Choice," American Psychologist, vol. 58, no. 9, pp. 697-720, 2003.

[11] D. Kahneman, Thinking, fast and slow. New York, USA: Farrar, Straus and Giroux, 2011.

[12] J. Heinström, "Five personality dimensions and their influence on information behaviour," Information Research, vol. 9, no. 1, pp. 1-24, 2003.

[13] G. Loewenstein and J. S. Lerner, "The role of affect in decision making," Handbook of affective science, vol. 619, no. 642, p. 3, 2003.

[14] J. S. Lerner, Y. Li, P. Valdesolo, and K. S. Kassam, "Emotion and Decision Making," Annual Review of Psychology, vol. 66, no. 1, pp. 799-823, 2015.

[15] M. L. Finucane, A. Alhakami, P. Slovic, and S. M. Johnson, "The affect heuristic in judgments of risks and benefits," Journal of Behavioral Decision Making, vol. 13 , no. 1, pp. 1-17, 2000.

[16] P. Slovic, M. L. Finucane, E. Peters, and D. G. MacGregor, "Rational actors or rational fools: Implications of the effects heuristic for behavioral economics," Journal of Socio-Economics, vol. 31, no. 4, pp. 329-342, 2002.

[17] G. Loewenstein, C. K. Hsee, E. U. Weber, and N. Welch, "Risk as Feelings.," Psychological Bulletin, vol. 127, no. 2, pp. 267-286, 2001.

[18] P. Slovic, M. L. Finucane, E. Peters, and D. G. MacGregor, "Risk as analysis and risk as feelings," Risk Analysis, vol. 24, no. 2, pp. 311-322, 2004.

[19] G. L. Clore and J. R. Huntsinger, "A Reply to Commentaries on "How the Object of Affect Guides its Impact"," Emotion Review, vol. 1, no. 1, pp. 58-59, 2009.

[20] J. H. Smith, T. Dinev, and H. Xu, "Information Privacy Research: An Interdisciplinary Review," MIS Quarterly, vol. 35, no. December, pp. 989-1015, 2011. 
[21] T. Dinev, A. R. Mcconnell, and H. J. Smith, "Informing Privacy Research Through Information Systems, Psychology, and Behavioral Economics: Thinking Outside the "APCO" Box," Information Systems Research, vol. 26, no. 4, pp. 639-655, 2015.

[22] F. Kehr, T. Kowatsch, D. Wentzel, and E. Fleisch, "Blissfully ignorant: the effects of general privacy concerns, general institutional trust, and affect in the privacy calculus," Information Systems Journal, vol. 25, no. 6, pp. 607-635, 2015.

[23] H. Li, R. Sarathy, and H. Xu, "The role of affect and cognition on online consumers' decision to disclose personal information to unfamiliar online vendors," Decision Support Systems, vol. 51, no. 3, pp. 434-445, 2011.

[24] K. P. L. Coopamootoo and T. Groß, "Why privacy is all but forgotten," No. 4, pp. 97-118, De Gruyter Open, 2017.

[25] L. Coventry, D. Jeske, and P. Briggs, "Perceptions and actions: Combining privacy and risk perceptions to better understand user behaviour," 2014

[26] S. Egelman, "My profile is my password, verify me!: The privacy/convenience tradeoff of facebook connect," in Proceedings of the SIGCHI Conference on Human Factors in Computing Systems, pp. 2369-2378, ACM, 2013.

[27] H. Krasnova, E. Kolesnikova, and O. Guenther, "'It Won't Happen To Me!": Self-Disclosure in Online Social Networks," in AMCIS 2009 Proceedings, p. 343, 2009.

[28] A. Raij, A. Ghosh, S. Kumar, and M. Srivastava, "Privacy risks emerging from the adoption of innocuous wearable sensors in the mobile environment," in Proceedings of the SIGCHI Conference on Human Factors in Computing Systems, pp. 11-20, 2011.

[29] S. Trepte, T. Dienlin, and L. Reinecke, Risky behaviors: How online experiences influence privacy behaviors, pp. 225-244. Munchen, DE: UVK, 2014.

[30] Y. Shulman, "Towards a broadening of privacy decision-making models: The use of cognitive architectures," in IFIP International Summer School on Privacy and Identity Management, pp. 187-204, 2017.

[31] I. Bilogrevic and M. Ortlieb, "'if you put all the pieces together...": Attitudes towards data combination and sharing across services and companies," in Proceedings of the 2016 CHI Conference on Human Factors in Computing Systems, pp. 5215-5227, 2016.

[32] A. P. Felt, S. Egelman, and D. Wagner, "I've got 99 problems, but vibration ain't one: A survey of smartphone users' concerns," in Proceedings of the Second ACM Workshop on Security and Privacy in Smartphones and Mobile Devices, pp. 33-44, 2012.

[33] A. Acquisti, L. Brandimarte, and G. Loewenstein, "Privacy and Human Behavior in the Age of Information," Science, vol. 347, no. 6221, pp. 509-514, 2015.

[34] M. Kezer, B. Sevi, Z. Cemalcilar, and L. Baruh, "Age differences in privacy attitudes, literacy and privacy management on facebook," Cyberpsychology: Journal of Psychosocial Research on Cyberspace, vol. 10, 052016.

[35] C. L. Miltgen and D. Peyrat-Guillard, "Cultural and generational influences on privacy concerns: a qualitative study in seven European countries," European Journal of Information Systems, vol. 23, no. 2, pp. 103-125, 2014.
[36] I. Junglas and C. Spitzmüller, "Personality traits and privacy perceptions: An empirical study in the context of location-based services," in International Conference on Mobile Business, ICMB 2006, pp. 387-402, 2006.

[37] E. E. Hollenbaugh and A. L. Ferris, "Facebook self-disclosure: Examining the role of traits, social cohesion, and motives," Computers in Human Behavior, vol. 30, pp. 50-58, 2014 .

[38] S. Egelman and E. Peer, "Predicting privacy and security attitudes," Computers and Society: The Newsletter of $A C M$ SIGCAS, vol. 45, no. 1, pp. 22-28, 2015.

[39] I. B. Weiner and R. L. Greene, Handbook of personality assessment. New Jersey, USA: John Wiley \& Sons, 2017.

[40] D. J. Leiner, "Too fast, too straight, too weird: Post hoc identification of meaningless data in internet surveys," 2013.

[41] M. B. Donnellan, F. L. Oswald, B. M. Baird, and R. E. Lucas, "The Mini-IPIP Scales: Tiny-yet-effective measures of the Big Five Factors of Personality.," Psychological Assessment, vol. 18, no. 2, pp. 192-203, 2006.

[42] A. J. Cooper, L. D. Smillie, and P. J. Corr, "A confirmatory factor analysis of the Mini-IPIP five-factor model personality scale," Personality and Individual Differences, vol. 48, no. 5, pp. 688-691, 2010.

[43] M. Tavakol and R. Dennick, "Making sense of Cronbach's alpha," International journal of medical education, vol. 2, pp. 53-55, 2011.

[44] H. C. Lench, S. A. Flores, and S. W. Bench, "Discrete emotions predict changes in cognition, judgment, experience, behavior, and physiology: A meta-analysis of experimental emotion elicitations.," Psychological Bulletin, vol. 137, no. 5, pp. 834-855, 2011.

[45] J. J. Gross and R. W. Levenson, "Emotion Elicitation using Films," Cognition and Emotion, vol. 9, no. 1, pp. $87-108,1995$

[46] M. K. Uhrig, N. Trautmann, U. Baumgärtner, R.-D. Treede, F. Henrich, W. Hiller, and S. Marschall, "Emotion Elicitation: A Comparison of Pictures and Films," Frontiers in Psychology, vol. 7, no. February, pp. 1-12, 2016.

[47] A. C. Samson, S. D. Kreibig, B. Soderstrom, A. A. Wade, and J. J. Gross, "Eliciting positive, negative and mixed emotional states: A film library for affective scientists," Cognition and Emotion, vol. 30, no. 5, pp. 827-856, 2016.

[48] A. N. Joinson, C. Paine, T. Buchanan, and U. D. Reips, "Measuring self-disclosure online: Blurring and non-response to sensitive items in web-based surveys," Computers in Human Behavior, vol. 24, no. 5, pp. 2158-2171, 2008.

[49] E. G. Carmines and R. A. Zeller, Reliability and validity assessment, vol. 17. Sage publications, 1979.

[50] A. Kitkowska, E. Wästlund, J. Meyer, and L. A. Martucci, "Is it harmful? re-examining privacy concerns," in IFIP International Summer School on Privacy and Identity Management, pp. 59-75, 2017. 